\title{
Programa de Atención Psicológica para los alumnos de la Facultad de Medicina de la Universidad de Barcelona
}

\author{
Psychological Care Program for the students of the Medical School of \\ the University of Barcelona.
}

Rosa Sender Romeo y Manel Salamero Baró

Facultad de Medicina. Universidad de Barcelona.

\begin{abstract}
El Programa d 'Atenció Psicològica de la Facultad de Medicina de Barcelona se desarrolló entre los años 1999 y 2003. Se razonan los motivos de su creación así como los aspectos específicos de pertenencia a una facultad de medicina. Se da cuenta de los trabajos de tipo asistencial realizados en el transcurso de ese periodo, así como de los trabajos de investigación correspondientes a la misma etapa o derivados de las líneas generales que se prolongaron con posterioridad al cierre del programa y que tienen su origen en la línea de trabajo del propio programa. Se analiza la conveniencia y el interés que puede atribuirse a este tipo de colaboraciones que pretenden valorar la etapa universitaria de los sujetos como la antesala de la vida laboral.
\end{abstract}

Palabras clave: estudiantes de medicina; programa de atención psicológica; ansiedad frente a exámenes; habilidades de comunicación.
Between 1999 and 2003 the program of the Barcelona UB Medical School Psychological Service was offered to the students. In this paper the reasons of its birth and specific linkage to the medical school are explained. An account is offered about the psychiatric and psychological are activity developed during this period, as well as the research projects which began during this time and were followed after the interruption of the care program. Finally the interest and convenience of these kind of programs are discussed, emphasizing the utility of considering the time spended at the university as the prelude of working life.

Key words: Medical students, psychiatric and psychological care program, exam anxiety, communication skills.

\footnotetext{
Correspondencia:

Rosa Sender Romeo.

Balmes 107, pral $1^{\mathrm{a}}$

Barcelona 08008

e.mail :6194rsr@comb.cat

Manel Salamero Baró

Facultat de Medicina de la Universitat de Barcelona

C/ Casanova, 143

08036 Barcelona

e.mail salamero@clinic.ub.es
} 


\section{INTRODUCCIÓN}

En el año 2001, se publicó en Educación Médica un artículo titulado El Programa de Atención Psicológica de la Facultad de Medicina de la UB ${ }^{1}$. En él se daba a conocer la existencia de un modesto equipo de trabajo recién fundado, se justificaba su función y se daba cuenta de sus proyectos. Era básicamente una declaración de intenciones, acompañada de unos primeros datos de tipo descriptivo recogidos los meses anteriores. El motivo de la creación del Programa d'Atenció Psicològica dels Estudiants de Medicina (PAPEM) puede resumirse en tres aspectos básicos.

De forma inmediata se partía del deseo de dar salida a las demandas que los estudiantes formulan para orientar sus problemáticas psicológicas. Por motivos razonables estas demandas tienden a canalizarse hacia los profesores de las asignaturas de psicología y psiquiatría, que coinciden con los años de inicio y finalización de la carrera y que suele ser etapas, tanto en nuestra facultad como en otras culturas, ${ }^{2}$ en las que hacen eclosión problemas adaptativos que pueden acompañarse en ocasiones de patología psiquiátrica. La dificultad que implica la derivación eficaz de estas demandas a través de la red pública, congestionada y con escasos recursos para tratamientos de tipo psicológico, fue un impulsor del proyecto.

Un segundo motivo pretendía enfrentarse al recelo que la práctica psiquiátrica ha despertado tradicionalmente en las otras especialidades médicas. Recelo en parte bien merecido debido a sus polifacéticas tendencias y su escaso interés por los aspectos evaluativos. Durante décadas esta especialidad se ha caracterizado más por su pintoresquismo que por su eficacia y, pese a que las circunstancias actuales son muy distintas, ya que nadie discute la presencia de variables psicológicas en el enfermar de cualquier sujeto y la investigación y los avances en genética, bioquímica y neuropsicología constituyen ámbitos de investigación punta, la práctica psiquiátrica sigue despertando más recelo del que merece comparativamente con otras especialidades. Datos de una encuesta llevada a cabo en la propia facultad de Barcelona ${ }^{3}$ hablaban de un porcentaje alarmante de utilización de medicinas alternativas por parte del alumnado, lo que presuponía una ignorancia de los modelos biomédicos aprendidos en el aula a la hora de orientarse en la búsqueda de ayuda. Es sabido que el fenómeno es aun más evidente cuando la demanda se refiere al malestar psicológico, en donde la oferta de soluciones alternativas alcanza lo prodigioso. De ahí la importancia que se concedió a que los estudiantes tuvieran acceso a una atención psicológica-psiquiátrica esmerada y competente que, a la par que resolviera sus problemas, les ofreciera un modelo sólido de intervención psiquiátrica. Se partió de la idea de que si los estudiantes, como futuros médicos, asimilaban con objetividad y rigor la importancia de la vida psíquica en el mantenimiento de la salud, se contribuía a convertirlos en profesionales aptos para manejar eficazmente estos conceptos en sus propios pacientes.

El tercer objetivo, relacionado con el anterior, pretendió contribuir a eliminar el estigma que pesa sobre el enfermo psiquiátrico. El temor a ser visto por los compañeros como paciente psiquiátrico se inicia tempranamente y persiste más de lo deseado. La preocupación por situar los servicios de atención a los estudiantes en lugares remotos y ocultos fue en su día uno de los temas reiterativos del II Encuentro Nacional de Servicios de Atención al Estudiante ${ }^{4}$. Un reciente trabajo analiza este mismo problema en universidades australianas ${ }^{5}$. Aceptar que cualquier ciudadano puede ser un usuario circunstancial de atención psicológica-psiquiátrica., nada distinto a los datos sobre salud pública que se manejan en toda Europa ${ }^{6}$ nos pareció una ayuda inmediata para su propia autoestima y para su futura relación con los pacientes. Por tal motivo la sede del PAPEM se anunció con la misma tipología grafica que cualquier otro servicio complementario dentro de la facultad y los programas de ayuda se expusieron abiertamente al alumnado en sus propias aulas.

\section{CARACTERÍSTICAS ESPECÍFICAS DE LA FACULTAD DE MEDICINA}

$\mathrm{Al}$ margen de los argumentos esgrimidos como punto de partida del proyecto, se tuvieron en cuenta los aspectos de esta facultad de medicina que le confieren una especificidad frente a otros Servicios de Atención Psicológica ya existentes en otras universidades españolas.

Selección del alumnado. Conviene puntualizar que los alumnos que acceden a la Facultad de Medicina de la Universidad de Barcelona han superado una elevada nota de selectividad. Este hecho determina que se trate de una población estudiantil que ya ha demostrado tempranamente unas técnicas de trabajo rentables. Asimismo, los datos presentados por el equipo decanal de la Facultad ${ }^{7}$ dan muestras de aprovechamiento a lo largo de la carrera y, en consecuencia, el abandono universitario es escaso. Estos datos positivos implican a su vez un alto grado de 
autoexigencia y cierta tendencia a la competitividad, aspectos ambos que pueden hacer dificultosa la adaptación de algunos estudiantes cuando pretenden llevar el mismo control exhaustivo sobre las materias académicas que llevaban en sus anteriores estudios.

Las demandas académicas, los horarios y especialmente los exámenes, actúan como estresores que pueden desencadenar estados de ansiedad y alteraciones del humor si el alumno carece de recursos para organizarse de forma adecuada. Bajo esa presión competitiva se interpreta como incapacidad intelectual lo que en realidad no es más que una incapacidad organizativa o un exceso de celo. Hablamos de un problema que más que nuevo es no resuelto, ya que hay abundante literatura anglosajona en las dos últimas décadas que señala las consecuencias del fenómeno y apunta algunas soluciones ${ }^{8,9}$

Aspectos preventivos del proyecto. El futuro de estos estudiantes al abandonar la facultad pasa por la preparación del examen MIR. Esto implica un árido esfuerzo de estudio, descontextualizado de la vida académica y del contacto clínico. Se trata de mantenerse durante meses sujeto a una disciplina solitaria a fin de poder competir con los colegas en un examen teórico. El buen temple emocional y la capacidad organizativa son los auténticos protagonistas del desafío.

Finalizada la etapa de preparación MIR, se inicia la vida laboral. Es sabida la elevada prevalencia de alteraciones psiquiátricas en los médicos, atribuidas fundamentalmente al estrés que supone la práctica asistencial. El Col-legi de Metges de Barcelona ya puso en marcha en 1999 un Programa d'Atenció Integral al Metge Malalt (PAIMM) que ofrece atención psicológica y psiquiátrica a los médicos. Partieron básicamente de un modelo teórico de atención a la toxicomanía, pero hubo que ampliar la ayuda psiquiátrica ya que la comorbilidad que subyace a las dependencias es muy alta ${ }^{10}$. El PAPEM se propuso ofrecer atención a los alumnos que lo solicitaran, pero también investigar en los perfiles de riesgo dentro del colectivo, con una intención claramente preventiva ya que está confirmada la dificultad que le supone al médico asumir el rol de paciente, al margen de la penalización que nuestra sociedad impone al enfermo psiquiátrico. Si tenemos presente que los actuales alumnos son los futuros médicos de nuestra sociedad, su paso por la facultad puede constituir un buen momento para aprender, no solamente las materias académicas, sino también el buen manejo de sus recursos psicológicos frente a situaciones estresantes ${ }^{11}$. El conocimiento de nuestra vulnerabili- dad, lejos de ser un oprobio, puede constituir una saludable información para prevenir conflictos innecesarios que repercutan en la futura práctica clínica. Esta actitud es ya habitual en las facultades del mundo anglosajón en las que se tienen en cuenta los costos invertidos en la formación del alumnado, lo que hace que sea importante la detección precoz de los sujetos que precisan ayuda o que se estima que serán incapaces de mantener una buena relación médico-paciente ${ }^{12,13}$

Feminización del alumnado. La entrada de la mujer como profesional de la medicina es en estos momentos un fenómeno totalmente asimilado en la cultura occidental. Algunas de sus consecuencias se van evidenciando, tanto en el ámbito del ejercicio de la profesión, como en el abordaje de la mujer como paciente. Los trabajos sobre esta cuestión son múltiples en los últimos años y también han trascendido al terreno de la educación médica ${ }^{14}$. La progresiva feminización del alumnado en nuestro país alcanza en los últimos años cifras entre el 60 y el $80 \%$. La incorporación gradual al medio laboral a lo largo de un par de décadas ha permitido una buena integración en el tejido social.

Los datos recogidos en una muestra de alumnas de último año, en la facultad de medicina de Barcelona, arrojan una mayor presencia de síntomas específicos de ansiedad y de síntomas generales de malestar psicológico ${ }^{15}$ en relación a sus compañeros. Cabe aventurar que en el futuro los compromisos profesionales de estas mujeres van a ser equivalentes a los de los varones mientras que no hay garantías de que se de la misma igualdad en lo tocante a otro tipo de responsabilidades. La reproducción y la crianza se siguen confiando básicamente a las mujeres. Será interesante ver si la muestra que actualmente atiende el PAIMM se desplazará hacia una demanda femenina y una patología más sesgada hacia los trastornos afectivos y las adicciones a benzodiazepinas como señalan los estudios epidemiológicos de género. No obstante, se sabe por múltiples trabajos, y esta muestra lo confirma, que las mujeres son más demandantes de atención sanitaria y tienen menos reparos para solicitar ayuda psicológica ${ }^{15}$

La variable género se tuvo muy presente al diseñar programas de intervención o de investigación en los trabajos del PAPEM. 


\section{ACTIVIDAD DEL PROGRAMA DE ATENCIÓN PSICOLÓGICA A LO LARGO DE SUS CUATRO AÑOS DE EXISTENCIA}

\section{ACTIVIDAD ASISTENCIAL.}

La mayoría de las demandas se llevaron a cabo entre los estudiantes con problemas de adaptación a la Facultad en los dos primeros cursos, y los que al finalizar la carrera se veían desbordados ante la experiencia del examen MIR.

Las dificultades académicas en los primeros cursos, seguidas de las familiares figuraron entre los estresores de mayor valor. Las épocas de exámenes y de recogida de resultados constituyeron los periodos punta de consulta. El tema, como viene reflejandose en múltiples trabajos ${ }^{16,17,18}$, ha sido investigado desde todos los puntos de vista

La preocupación excesiva por el peso, sin llegar a reunir necesariamente criterios de trastorno alimentario, se agudizaba en épocas de exámenes dando lugar a episodios de atracones con frecuentes demanda de ayuda.

Se abrieron 140 historias clínicas.

Se precisó de tratamiento farmacológico para un $60 \%$ de los alumnos y fueron derivados a un especialista del dispensario del Institut de Psiquiatría del Hospital Clínic con quien se estableció un acuerdo.

Para subsanar deficiencias en los hábitos de trabajo o dudas respecto a la orientación profesional se estableció una colaboración con el Gabinet d'Orientació Universitaria, que resultó fluida y eficaz desde un principio.

Los trastornos alimentarios de gravedad, incluyendo un ingreso, se negociaron con el Institut de Trastorns Alimentaris (ITA) que proporcionó una cobertura económica total a través del seguro escolar.

\section{Atención psicoeducativa en grupos.}

Desde el PAPEM se ofreció a los estudiantes de la facultad programas a modo de talleres psico-educativos en los que se trabajaron recursos para facilitar su solvencia y reducir el malestar asociado a aspectos propiamente académicos:

Programa para afrontar la ansiedad ante los exámenes; taller psico-educativo dirigido a los estudiantes que manejaban con dificultad el estrés asociado al periodo de evaluación y que veian afectado su rendimiento académico y/o su equilibrio psíquico. Este taller se desarrollaba en 9 sesiones en las que se trabajaban distintos recursos y estrategias cognitivoconductuales para afrontar de modo eficaz el manejo de la ansiedad. Se llevaron a cabo en los dos últimos años de funcionamiento del PAPEM mediante dos convocatorias anuales coincidiendo con las semanas anteriores a los exámenes de febrero y junio. Acudieron en su mayoría alumnos de los dos primeros cursos. Se dió cuenta de los resultados en esta misma publicación. ${ }^{19}$

Programa para el control de la ansiedad y la mejora del rendimiento académico de los alumnos que preparan el MIR; taller psico-educativo dirigido a los alumnos de $6^{\circ}$ curso que habían superado las materias y afrontaban la preparación del examen MIR. Con esta actividad se pretendía un doble objetivo: fomentar una organización efectiva del trabajo de preparación del examen y promover el aprendizaje de herramientas que permitieran manejar el estrés y la ansiedad que implica la preparación del MIR20. Como ya se dijo, se trata de un periodo especialmente ansiógeno, debido a la significación personal que el examen MIR tiene para los alumnos y a las características diferenciales de esta evaluación respecto a los exámenes de la licenciatura, en cuanto a la cantidad de contendios y la estrategia en la organización de un periodo de estudio a largo plazo. Los talleres constaron de 12 sesiones, con un máximo de 10 alumnos por grupo y estos grupos se repartían entre junio y setiembre.

El número de alumnos atendidos a lo largo de estos cuatro años fue de 124. Los programas se fueron remodelando en función de las evaluaciones de los usuarios. La impresión de prestar un servicio eficaz y valorado vino refrendada por el éxito de las convocatorias.

\section{ACTIVIDAD CIENTÍFICA INVESTIGADORA}

El estudio de los factores de vulnerabilidad de esta población constituyó un aspecto esencial del Programa. Interesaba saber qué variables contribuían a que algunos fueran más sensibles a las situaciones estresantes derivadas, por el momento de la demanda académica, y en el futuro, de la profesional.

Desde su creación el PAPEM llevó a cabo una serie de trabajos epidemiológicos encaminados a detectar la presencia de psicopatología temprana en esta muestra de población. Los resultados se han dado a conocer en diferentes foros científicos ${ }^{21,22,23}$. Estos resultados confirman la presencia de mayores porcentajes de sintomatología psiquiátrica, comparada con otros colectivos Actualmente disponemos de los datos de seguimiento de una cohorte evaluada en primero y en sexto año académico, justo antes de entrar en la vida laboral. De esta última evaluación, reali- 
zada tras el cierre del PAPEM se han dado a conocer algunos datos ${ }^{15}$.

El último tema de investigación para el que se dispuso de financiación (ayuda del Departament d Universitats Reserca i Societat de l'Informació), se realizó también tras el cierre asistencial del PAPEM, y consistió en un análisis de las habilidades de comunicación, adquiridas por los estudiantes de nuestra facultad en el último año de carrera, aplicadas a la entrevista clínica. Al comenzar a valorarse la posibilidad de que este tipo de habilidades formaran parte en un futuro de la selección para los MIR se creyó interesante analizar el estado actual de la cuestión en el alumnado. Es sabido que gran parte del malestar de los profesionales de la salud está vinculado a las dificultades en la relación médico- paciente ${ }^{24,25,26}$. Las Facultades de Medicina hasta ahora han basado sus enseñanzas en los conceptos teórico- prácticos sin a penas tener presente la importancia de una interacción eficaz que garantice la utilidad del acto médico. Los resultados son elocuentes y están pendientes de publicación ${ }^{27}$.

\section{Valoración al final del programa}

El programa se cerró a los cuatro años de su puesta en marcha por falta de financiación. En un momento en el que el proyecto había obtenido la aprobación de los alumnos parecía prudente asegurar su continuidad, tanto desde el punto de vista clínico como a la hora de solicitar ayudas para investigación, así como para establecer relación con otras instituciones interesadas.

El final del PAPEM dejó pendientes, entre otras, dos líneas de estudio que estimábamos de crucial interés: el profundizar en las demandas de las alumnas por trastornos alimentarios que se exacerbaban bajo estrés, y un estudio sobre el consumo de drogas a lo largo de los años de carrera. El modelo académico constituye un estresor relativamente homogéneo en el tiempo y en la demanda, lo que permite valorar el componente de vulnerabilidad personal, ya sea debido a la diátesis del sujeto o a las circunstancias biográficas por las que atraviesa en el periodo académico.

Es difícil de explicar que una experiencia que se mantuvo activa durante cuatro años no haya tenido mejor suerte y pueda haberse interpretado como un intento de sobreprotección a los estudiantes de medicina ofreciéndoles un producto que ya está en el mercado. Es de esperar que como no se trata de un concepto ni costoso ni único, en el futuro la mirada hacia el mundo anglosajón haga pensar que sería conveniente potenciar una estructura que fuera tomando el pulso psicológico a los alumnos. En realidad se trata de un elemento educativo más, como se evidenció en la evaluación externas de la facultad.

Hubo ocasión de comprobar esta realidad al cerrar el Programa y tener que enfrentarse a los alumnos que quedaban pendientes o que acudían en busca de información. En un tiempo en el que la autonomía de los centros permite, y aun exige, la noble competencia en la formación de los futuros profesionales, habría que ser capaz de ampliar la mirada más allá de la imprescindible tecnología, que se va resolviendo de forma excelente, y ser capaces de ver qué otros aspectos serán de utilidad al alumno cuando salga a ejercer su profesión ${ }^{28}$. Aportarle un bagaje de conocimientos para su propio cuidado psicológico y el de sus pacientes, no es una entelequia, ni un síntoma de debilidad. No es más que dar respuesta a una evidencia.

\section{BIBLIOGRAFÍA}

1. Sender R, Salamero M, Vallés A, Díaz L. El programa de atención psicológica de la facultad de medicina de Barcelona. Educ Méd 2001. 4 (2):66-71.

2. Miller McC. The first year at medical school: some findings and students perceptions. Med Educ. 1994, 28: 5-7

3. Vallés A, Forcades T. Los estudiantes de medicina y las medicinas alternativas. XV Congreso Nacional de la Sociedad Española de Educación Médica. Granada. 2001

4. Sender R. Creación y puesta a punto de un programa de atención psicológica a los estudiantes de la Facultad de medicina de la UB. II Encuentro Nacional de Servicios de Atención al Estudiante. Málaga. 2000

5. Brimstone R, Thitslethwaite J, and Quirk F. Behaviour of medical students in seeking mental and physical care: exploration and comparison with psychology students. Med Educ 2007; 41: 74-83.

6. Valdés M., de Pablo J, Campos R, Farré,J.M. et al. El proyecto multinacional europeo y multicéntrico español de mejora de calidad asistencial en psiquiatría de enlace en el hospital general: el perfil clínico en España. Med Clin (Barna) 115: 690-694. (2000)

7. Jornadas de Docencia de la Facultad de Medicina de Barcelona. Datos del equipo decanal. Octubre del 2000. Barcelona.

8. Coles C. Introduction: Medicine and Stress. Med. Educ.. 1994, 28: $3-4$

9. Wolf T.M. Stress, coping and health: enhancing well-being during medicak school. Med. Educ. 1994, 28: 8-17.

10.Arteman A. Informe sobre el primer any de funcionament del Programa d’Atenció Integral al Metge Malalt (PAIMM). Servei d’Informació Col.legial. Decembre- gener, 2000. 92: 19-21

11.Mosley T.H., Perrin S.G., Neral M.S., Dubbert P M., Grothues 
C.A. and Pinto Stress, coping and well-being Acad. Med. 1994. 69: 765-767.

12.R. Stephen Manuel, Nicole J. Borges, and Holly A. Gerzina. Personality and clinical skills: any correlation ?. Acad Med.80 (10): 530-533.October 2005 Supplement

13.Knights $\mathrm{J}$ and Kennedy B. Medical school selection: impact of dysfunctional tendencies on academic performance. Med Edu 2007: 362-368

14.Kilminster S, Downes J, Gough B, Murdoch-Eaton D and Robert T. Women in medicine- is there a problem ? A literature review of the chanching gender composition, structures and occupational cultures in medicine. Med Edu 2007: 41: 39-49.

15. Sender $\mathrm{R}$ Diferencias de género en población de estudiantes de medicina. 1er Congrés Català de Dona i Salut Mental. Barcelona 2006

16.De Pablo J., Subirá S., Martín M.J., De flores T y Valdés M. Examination-associated anxiety in students of Medicine. Acad. Med. 1990; 65: 706-707

17.Armario A., Marti O, Molina T., De Pablo J, Valdés M. Acute estress markers in humans: response of plasma glucose, cortisol and prolactin to two exams in the anxiety they provoke. Psychoneuroendocrinology. 1996; 21:17-24

18.Penzo W., Martínez A. y Vallés A. Es estressant estudiar medicina? Poster Jornadas de Docencia. Facultad de Medicina de la UB. Octubre de 2000. Barcelona.

19. Sender R. Vallés A. Puig O. Salamero M. Valdés M. ¿Qué hay detrás del miedo a los exámenes? Educ Med. 2004.7 (i): 36-41.

20.Diaz L, Vallés A, Sender R, Salamero M. Presentación y evaluación de un curso para aprender a controlar la ansiedad durante la preparación del examen MIR. Educ Méd. 2001. 4 (3): 119-119.

21. Salamero V Congreso Anual de Psiquiatría y Psiquiatría Biológica-Sociedad Española de Psiquiatría Biológica y Sociedad Española de Psiquiatría. Barcelona, del 5 al 9 de noviembre de 2002. Ponencia en Mesa de Debate: "Estudiar Medicina... ¿es un factor de riesgo para la salud mental?". Dr. Manel Salamero.

22. Salamero M., Sender R, Díaz L IV Jornadas de Docencia de la Facultat de Mecicina de la UB. Barcelona, 2000. Póster: "Análisis de Factores de Riesgo Psicológico en una Población de Primer Curso de Medicina de la UB".

23.Sender R, Salamero M, Vallés A, Valdés M. Psychological Variables for Identifying Susceptibility to Mental Disorder in Medical Studens of Barcelona University. 2004. Med Educ online 9.9:1-5

24.Firth-Cozens J. The Psychological problems of doctors. 79-92. In: Stress in Health professionals. Ed. Fith-Cozens J and Payne R. 1999. Wiley\&sons.England.

25.Moss. F, and Paice E. Getting things right for the doctor in training. En: Stress in Health Professionals. Edit J. Firth-Cozens and R. Payne. 1999. Wiley \& Sons. England. Pag:203-218.

26.Wagner P.G., Lentz L., Heslop S.D. Teaching communication Skills: a skill-based approach. Acad Med. 77 (11): 1164, 2002.

27.Sender R, Valdés M, Salamero M, Mitjanas TH, Vallés A: Personality variables and clinical interview in medical students who are about to graduate. Pendiente de publicación. 2007

28.Valdés, M. Crónica de una muerte aplazada. Revista de Psiquiatría de la Facultad de Medicina de la Universidad de Barcelona. 2006. 33 (2): 59-60 